\section{Autophagy: to die or not to die}

When extracellular nutrients or growth factors are limited, eukaryotic cells sequester cytoplasmic components into phagosome vesicles to be targeted for lysosomal degradation, in a process known as autophagy. It is unclear, however, whether cells use 'self-digestion' as a means of survival by providing an alternative, intracellular energy source or whether autophagy is yet another cell death pathway, distinct from apoptosis or necrosis. Levine and colleagues report (Cell 122, 927; 2005) that the anti-apoptotic protein Bcl-2 functions to limit autophagy to levels compatible with cell survival.

The authors showed that exogenous expression of cellular or viral Bcl-2 inhibits starvation-induced autophagy in both yeast and mammalian cells. In contrast, downregulation of $\mathrm{Bcl}-2$ increases formation of autophagososmes under these conditions. By crossing mice transgenically expressing Bcl-2 with mice expressing a fluorescently labelled autophagy marker, the authors observed that after prolonged starvation, the levels of autophagy were decreased in cardiomyocytes of Bcl-2 expressing mice compared with control mice, thus establishing a physiological role for $\mathrm{Bcl}-2$ in the regulation of this lysosomal pathway. Bcl-2 is an established regulator of apoptosis and even though additional functions have been attributed to Bcl-2 family members, its role in the lysosomal pathway may be another way that it controls cell homeostasis. Intriguingly, Bcl-2 seems to inhibit autophagy at the endoplasmic reticulum, rather than at mitochondria where it is known to regulate apoptosis. So how does Bcl-2 prevent autophagy at the ER?

Beclin-1 participates in the formation of autophagosomes and was identified as a Bcl-2-interacting partner. The authors found that Bcl-2 expression could rescue cells from Beclin-1-induced autophagy and that this required a physical interaction between the two proteins: Beclin-1 mutants defective in their ability to bind to Bcl-2, or Bcl-2 mutants defective in binding Beclin-1, blocked Bcl-2-mediated inhibition of autophagy. Furthermore, Beclin-1 mutants that cannot bind Bcl-2 induced autophagy-dependent cell-death and increased basal

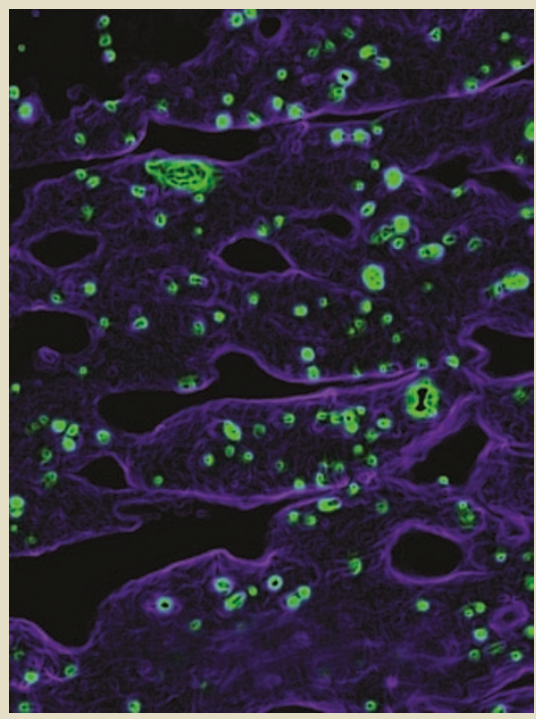

$\mathrm{Bcl}-2$ transgenic expression reduces the number of autophagosomes (green dots) in cardiac muscle cells. A light microscopic image of starved mouse heart is shown, transgenically expressing the fluorescently tagged autophagy protein marker GFP-LC3. Image reproduced from Pattingre et al. Cell 122, cover (2005), with permission from Elsevier (2005).

levels of autophagy even under conditions of normal growth. This could be blocked by short-interfering RNA (siRNA)-mediated downregulation of the downstream autophagy gene, atg5. Beclin-1's function in autophagy depends on its interaction with Class III PI(3)K but whether Bcl-2's inhibitory role is to disrupt the Beclin-1/Class III $\mathrm{PI}(3) \mathrm{K}$ interaction remains unclear.

Collectively, this work demonstrates that the Bcl-2-Beclin-1 interaction acts as a threshold to keep the levels of autophagy in check. This is further supported by the finding that Beclin-1 and Bcl-2 associate in a nutrient-dependent manner, whereby starvation decreases the amount of Beclin-1 bound to Bcl-2 and nutrient availability has the opposite effect. Future experiments will hopefully uncover the mechanism that tips the fragile balance of cell survival and cell death.

MYRTO RAFTOPOULOU 